\title{
Iot Based Health Care System for Predicting Cardiac Issues
}

\author{
A Chandrahas Reddy, G Avinash Reddy, A V R M Koushik, K V Siddeshwar, C Fancy
}

\begin{abstract}
According to the survey conducted by the WHO (World Health Organization), out of 56.9 million deaths, Ischemic heart disease and Heart stroke account for $\mathbf{1 5 . 2}$ million deaths of the total deaths in 2016. These are regarded, as the Non-Communicable Diseases (NCD) also known as chronic diseases, tend to affect a person for a long duration. Along with in most of the cases, it is hard to find the disease existence in its primary stage; we can find it only with the symptoms like stroke or heart attack. Due to the lack of these symptoms, healthcare awareness and the financial needs many people are losing their lives. It is a very long process and cost effective. Hence, we are proposing a model, which predicts these symptoms with the implementation of latest technological advances like IoT, machine learning and deep learning algorithms. The proposed methodology takes place in three stages. Primary stage consists of collecting the data through sensors attached to the patient. Secondary stage involves the transfer of data from the microcontroller to the application and converting the data to suitable form. Tertiary stage involves feature extraction of the data and classification of the data using the CNN algorithm. All the stages run dynamically and generate the results based on the data collected. These results are then analyzed to check if the patient has arrhythmias or normal.

Keywords: ECG, IoT, CNN, Neurokit
\end{abstract}

\section{INTRODUCTION}

In today's competitive world people has many responsibilities to look after and manage. This has become quite common especially for the people who do the job that involves many tasks of unique kind and different challenges daily. This led the people to shift their focus onto work, which made them avoid taking care of their health activities. This leads them to feel increased pressure and stress over them, get expose to many ill habits like smoking, excessive use of alcohol and caffeine, unnecessary medical prescriptions to counter medical issues and get ill health situations like diabetes, high blood pressure and the deadly cardiac issue. Taking a good care of health issues and having an awareness about the health condition helps us to counter

Revised Manuscript Received on April 25, 2020.

* Correspondence Author

A Chandrahas Reddy*, Pursuing B.Tech, Department of Information Technology, SRM Institute of Science and Technology, Chennai.

G Avinash Reddy, Pursuing B.Tech, Department of Information Technology, SRM Institute of Science and Technology. Chennai.

A V R M Koushik, Pursuing B.Tech, Department of Information Technology, SRM Institute of Science and Technology. Chennai.

K V Siddeshwar, Pursuing B.Tech, Department of Information Technology, SRM Institute of Science and Technology. Chennai.

C Fancy, Assistant Professor, Department of Information Technology, SRM Institute of Science and Technology. Chennai.

(C) The Authors. Published by Blue Eyes Intelligence Engineering and Sciences Publication (BEIESP). This is an open access article under the CC BY-NC-ND license (http://creativecommons.org/licenses/by-nc-nd/4.0/) these ill health situations. By a survey that has been conducted by WHO about 15.2 million deaths out of 56.9 million deaths in 2016 are caused by Ischemic heart disease and Heart stroke, which alarms the world that a necessary step needs to be taken. Any problem in our life, can be solved by knowing its root cause and the ways that the problem has surfaced. The problem can be solved by understanding, learning and analyzing the cause of the situations to arise. That is the reason why we need to take proper care and immediate action for any random problem we face in our daily life. All the concepts have some solution in daily life, but the solution lies in the way we treat the problem or how we treat or analyze the problem. Health of a being is something, which we need to monitor regularly. This can be done by following a diet, maintaining physical fitness, avoiding unhealthy and ill food, which cause strange diseases on consumption. The world has witnessed this recently in the form of COVID-19 (Corona Virus) which has its origin due to the adaption of strange consuming habits. This led the humankind to sacrifice thousands of people and made many lose their loved ones. The world is still suffering with this and affecting many people. Therefore, this developed project counters the problem of people not knowing their heart state, which is termed as Arrhythmia. Arrhythmia consists of various modes of heartbeat, which are forms of different irregular heartbeats. These different types of heartbeat motions are studied and gives the state of the heartbeat. Our project is a work that helps people to understand their health issues based on their mode of heart functioning and get them the necessary care they need if the situation is not in their favor. To make it possible to track the activity of the person regularly. This is feasible only by involving a dedicated hardware device that monitors the person's activity. This hardware device is developed on a microcontroller that has atleast one analog pin that acts as a pin to receive the data produced by the ECG sensor. This ECG sensor has ECG electrodes, which calculate the voltage difference produced due to the heart motion during the process of pumping the blood throughout the body. Later, this data collected by the microcontroller at the analog pin is understood and analyzed using the designed machine-learning algorithm. This algorithm gives results based on its study of the ECG values those generated [1]. These results are studied and result the type of heartbeat the person is having and helps us decide if the person need any medical assistance.
Published By:

Blue Eyes Intelligence Engineering \& Sciences Publication

(C) Copyright: All rights reserved. 


\section{RELATED WORK}

The existing model in real-time for tracking and recording of electric impulse produced by the motion of heartbeat is achieved only using the ECG, monitor which is present only in hospitals. This has led people to limit their need to track the electric impulse produced to track only in a hospital. The project that are proposed previously are only for any pre-recorded data for a patient.

They don't have any system or device to track their ECG values dynamically and obtain the results instantly. This has been a problem for the patient's by not letting them to have knowledge about their condition.

[1] This paper briefs about the IoT Service Platform Layer characterizes and gives perfect IoT organization reflections that can be used by various applications. There can be a course of action of stage organizations from the IoT arrange base. Further, a similar structure can be connected with application organizations where a bit of the reusable application parts is available as organizations.

[2] This paper proposed an IoT-based coronary illness observing framework for pervasive medicinal services administration. This framework screens the patients' physical signs just as significant ecological markers persistently, and gives four unique information transmission modes that parity the human services need and interest for correspondence and computing resources.

[3] In this paper the feature selection is been implemented in the matlab and the ECG segment is been processed with the cut-off frequency of $5 \mathrm{HZ}$. For this, they used the QRS wave detectors with the help of Pan-Tompkins algorithm and divided the signal processing into 10 -second wave segments. After that they implemented the deep learning algorithm especially the CNN which is the Convolution Neural Network because of its computational efficiency and the only drawback of using the $\mathrm{CNN}$ is that it functions on the grid like structure. While experimenting they used the 5-fold cross-validation to assess the performance of the complete proposed project.

[4] This paper gave a clear explanation about ECG signal examination and arrangement strategy utilizing wavelet vitality histogram technique and support vector machine (SVM). The characterization of cardiovascular coronary illness in the ECG signal comprises of three phases including ECG signal preprocessing, highlight extraction and pulses characterization.

[5] This paper gives a regular component determination technique for Heart Disease Prediction. The QRS indicator utilizing SVM is proposed and implemented to the CSE ECG database. The data about the QRS edifices acquired by this strategy is extremely valuable for ECG order and cardiovascular determination.

\section{Drawbacks:}

- Few of the models use Bluetooth to transfer the data which is more power consuming

- These systems have no feature of processing the data obtained from patient dynamically.

\section{PROPOSED MODEL}

In the proposed model, we use NODEMCU ESP32 microcontroller, AD8232 (Heart Rate Sensor), using these an IOT component is designed. Initially the device is configured and assigned to a patient. We connect three ECG electrodes on the patient's body (Right Arm, Left Arm, Right leg), it is generally regarded as Single Lead Electrode [5]. These electrodes are connected to the AD8232 sensor using a three cable snap which gives the analogue signal of voltage and collected at the sensor module through an audio jack. These data collected from the Sensors are then sent to a specifically dedicated port of the system. The data is then collected at this port and stored in the form of a CSV file. We use neurokit, that helps to extract the feature of many signals like ECG, EDA, RSP, EMG. This is a widely used feature extraction, integration of high-level complex statistical features and for bio-signal processing [2]. This neurokit, gives filtered data that can be used for processing the using CNN algorithm [3]. Then this data is classified into four categories, Arrhythmias, Normal, Other signal, negation. Each category intimates the type of signal the data contains and also describes the level of each category [4].

\section{Advantages:}

- The model employs both the neurokit and also the CNN algorithm

- This model involves the dynamic implementation of the whole process rather than implementing each step manually 


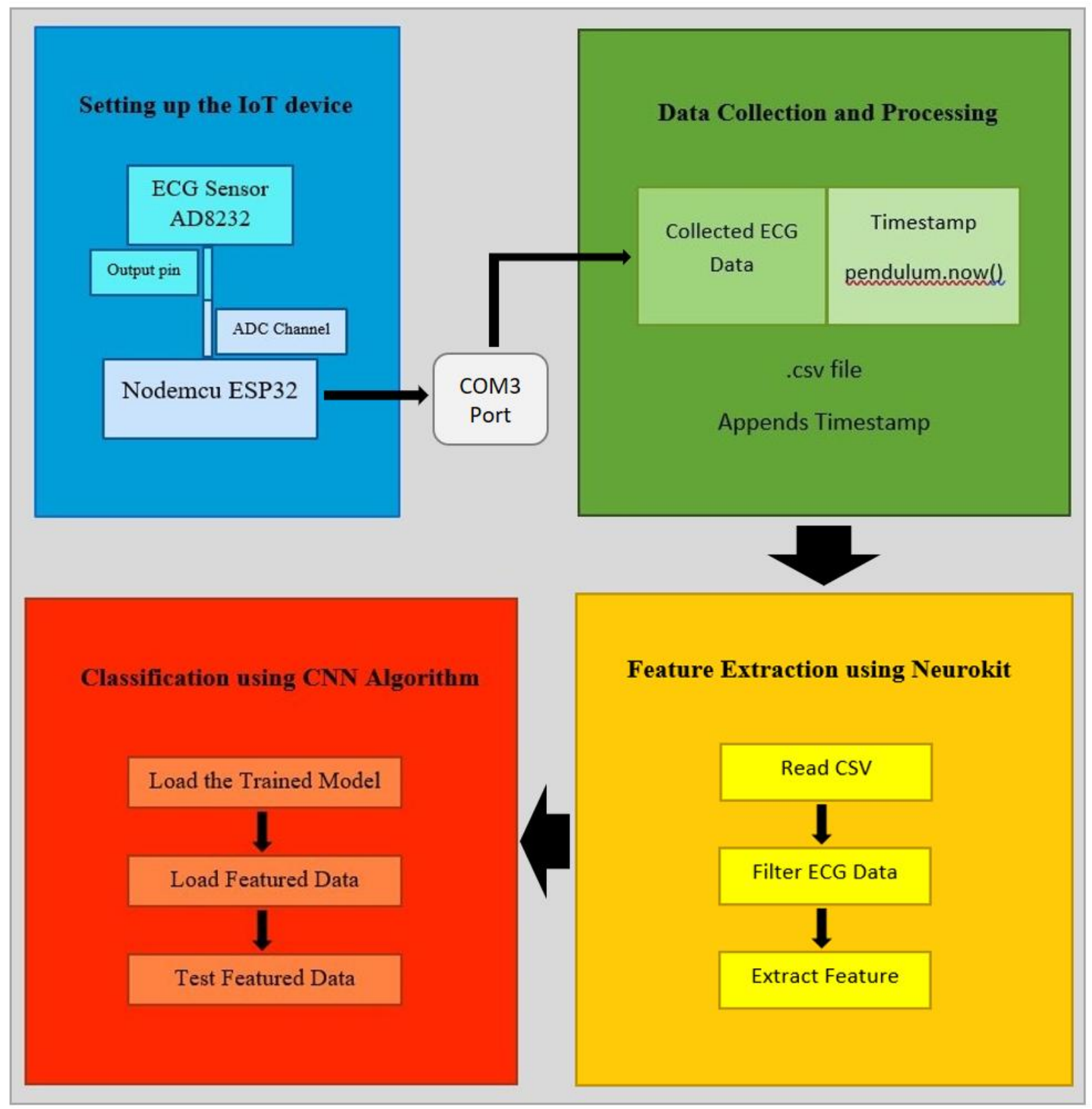

Fig 1. System Architecture

The modules the proposed system contain are:

A.Setting up the IoT device

B. Data Collection and Processing

C. Feature Extraction using Neurokit

D.ECG Classification using CNN Algorithm

A.Setting up the IoT device

This whole module revolves around dynamically processing the steps:

i. Collecting the electric activity of the patient produced due to the heartbeat of the patient. The electrodes should have v. adhesive which lasts longer and remains fresh throughout the process ii. The electrodes should be placed properly. The Red cable on the right side of the chest or on the right arm, the Yellow cable on the left side of the chest or on the left arm and finally the Green cable that acts as a neutral should be attached on the right leg or on the right side below the chest

iii. The data collected at the electrodes is then transferred to the microcontroller ESP32

iv. The data collected at the analog pin in the microcontroller is then sent to the COM3 port (communication port 3)

. This data acts as raw ECG signal data. The data collected at the port can be viewed parallel at Serial Monitor to view the values and the serial plotter to view in a graphical form

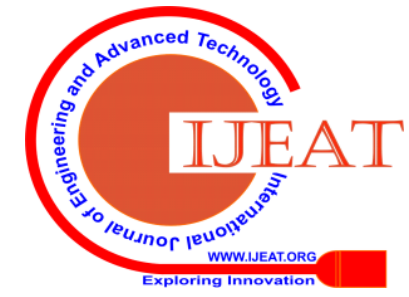




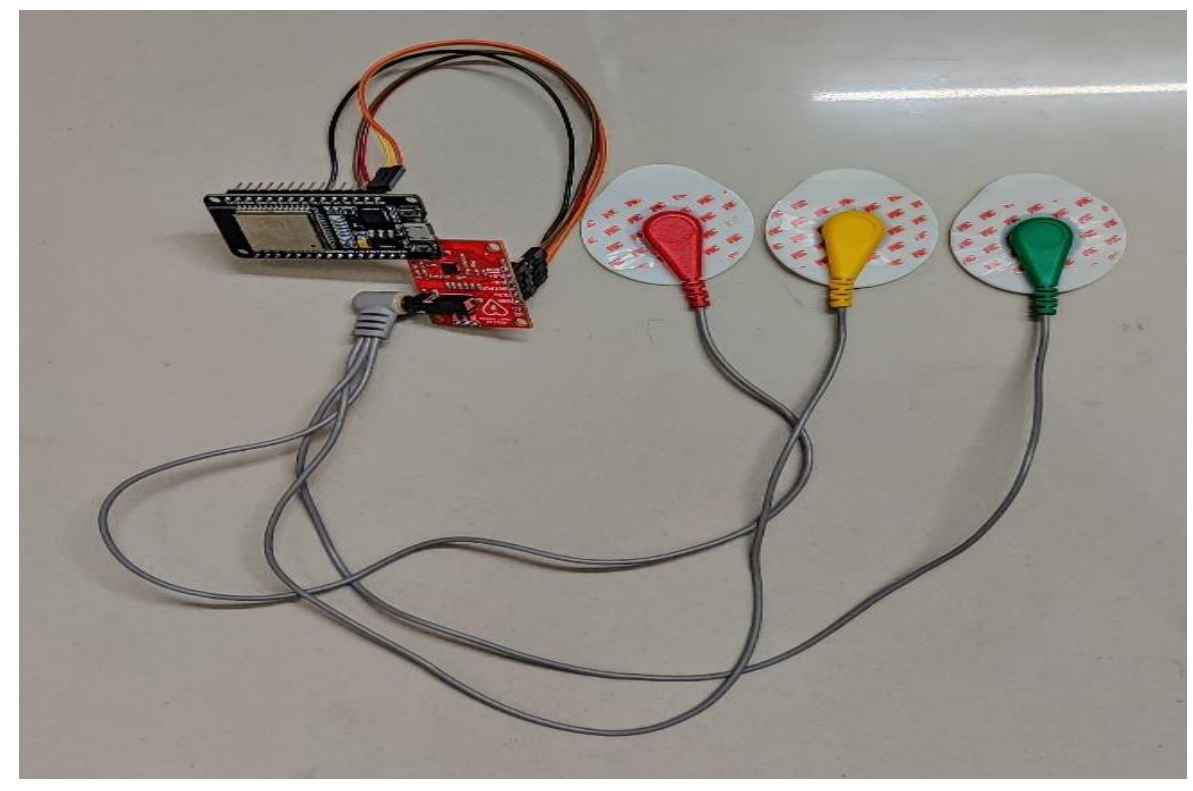

Figure 1 Assembled IoT Device

\section{B.Data Collection and Processing}

The steps this module includes:

$\mathrm{i}$. The data that is transferred to the port by microcontroller is collected by a python file

ii. This data collected is pasted in an Excel sheet and the timestamp when the data collected is also appended to the data

iii. Once the data is collected, the python file converts the Excel sheet into a CSV file

\section{C.Feature Extraction using Neurokit}

The steps this module involves are:

i.CSV file is loaded into the feature extraction module and its details are processed (determines the frequency for the timestamped data)

ii. Using neurokit.ecg_process() we filter and extract features of the ECG data

iii. By Plotting the filtered data based on its properties feature is studied and a CSV file is generated for the filtered data

iv. This CSV file is later used by the next module as its input

\section{D.ECG Classification using CNN Algorithm}

This module involves the process of classifying the feature extracted data from the machine learning algorithm. The signal generated is stored in the form

of an image and it is sent to process. It have four classes namely:
i. Class A - Atrial Fibrillation
ii. Class D - Disturbance
iii. Class O - Other Signals
iv. Class N - Normal Signal

The CNN algorithm stores the score of each class, displays the class with highest score and the share of the score from the data. The module involves the following steps in its processing:

i. Import the trained model that has the ability to test the data we provide dynamically

ii. The featured data in the form of CSV file in previous module is imported into this module

iii. With the help of imported model which have its own ways and methods for processing the data, tests this imported CSV file

iv. It displays the quality of the signal we provide and it dynamically determines the frequency of the data filtered

In the end, the class with highest score is displayed along with its share of recordings in the data provided

\section{ANALYSIS}

As we take live readings from the patient it is essential to record data with high quality. The sensor readings may contain noise in it. The cause of the noise may be either the movement of the muscles which produce EMG or if the laptop is connected to power supply DC interference may also cause the noise. So in order to get the data with high quality it is recommended to take the readings with the patient in rest position. The frequency of reading the data from the IOT module also determines the noise levels in the readings. We used a frequency of 330 readings per second. In the Figure 2.a, shows the distortion in the graph due to the improper connection of the ECG electrodes to the patient body. The Figure 2.b denotes is the data with min disturbance obtained when the patient is in rested position and we can also observe that the graph have minimal disturbances.

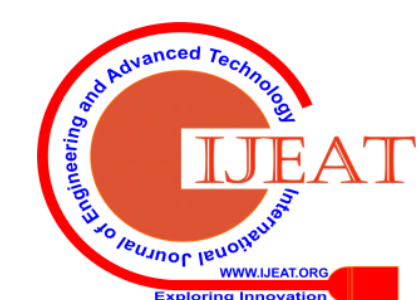



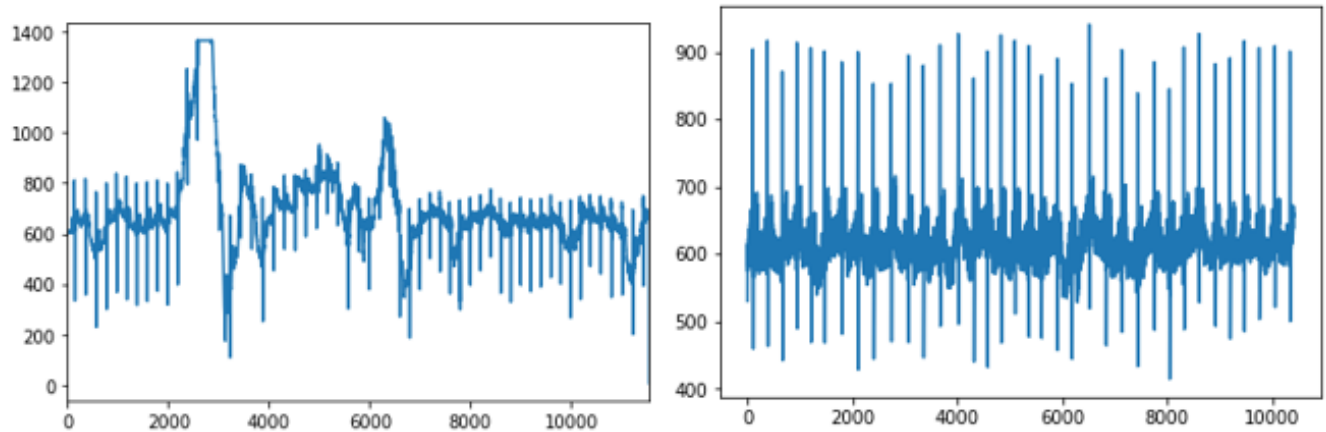

The data collected is then filtered using band pass filter to eliminate noise of high frequency. Filtering is a vital step which increases the quality of the signal before the feature extraction. We need to set the low and high frequency to allow
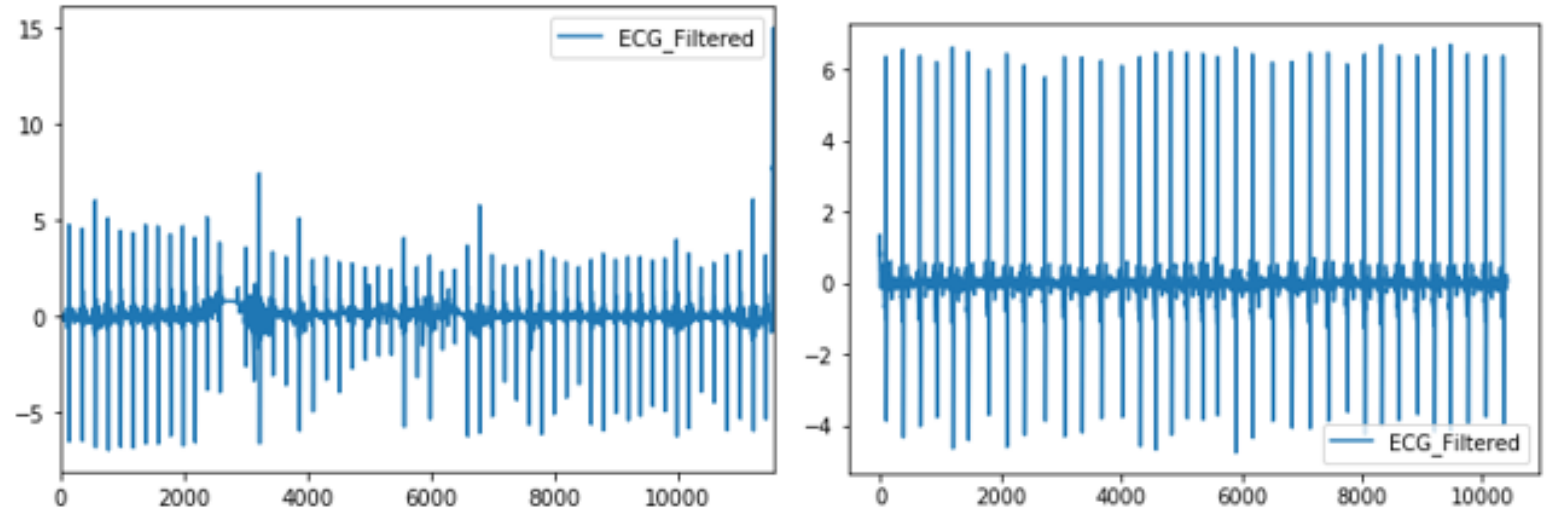

Figure 3 ECG Filtered Data a) When loosely connected b) When Person in Resting Position

Feature extraction is process of identifying the unique features from the data and extracting the information about the data using the values of these features. In this project we extract features like R_peaks, Cardiac_cycles, T_waves, P_waves, Q_waves, S_waves, T_waves_onset, Q_waves_onset, T_waves_ends, Probable_lead, Cardiac_Cycle_Signal_quality, Average_signal_Quality,

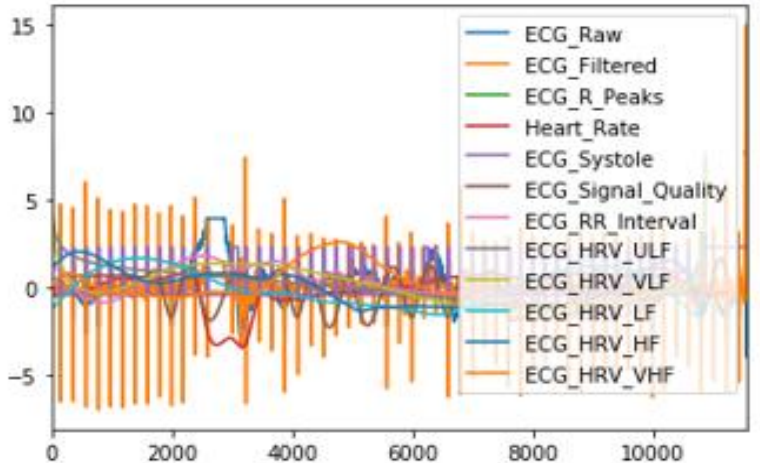

in the wave . This also aligns the wave form slightly in order to remove the noise in the AD8232 sensor due to the movement of chest due to respiration.

HRV, ECG_signal_Quality. Using the HRV features we classify the data based on the Physionet ECG shortlead data provided for the 2017 CINC challenge. In this dataset we are provided with total of 8528 records each ranging from 30 s to 61s ECG recordings of a single person. Data is classified into 4 classes Normal rhythm, Atrial Fibrillation, Noise or disturbance and other rhythm.

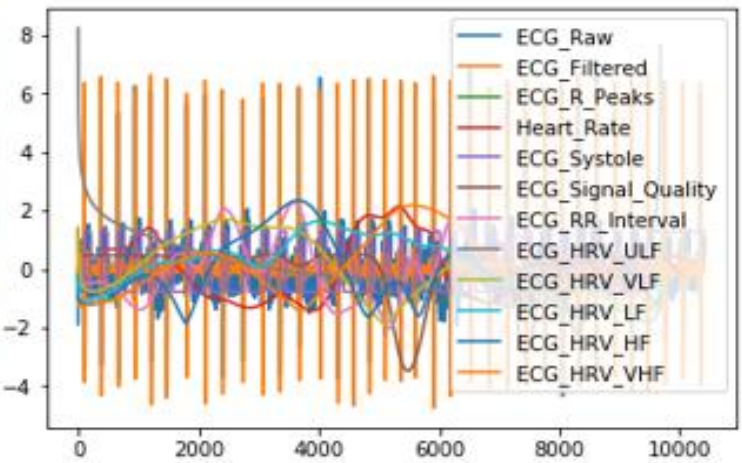

Figure 3 Features Extracted a) When loosely connected b) When Person in Resting Position

We determine the quality of the wave based on the Cardiac_Cycle_Signal_quality, Average_signal_Quality, ECG_signal_Quality. Average signal quality of the devise is around $83.4 \%$. Using CNN algorithm we determine the class of the reading with the certainity \% based on the score of the hidden layer score.

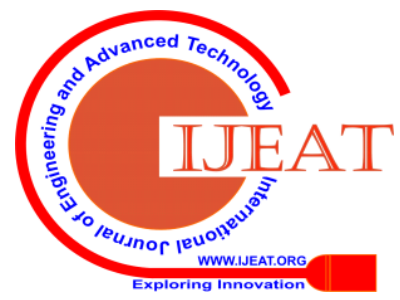



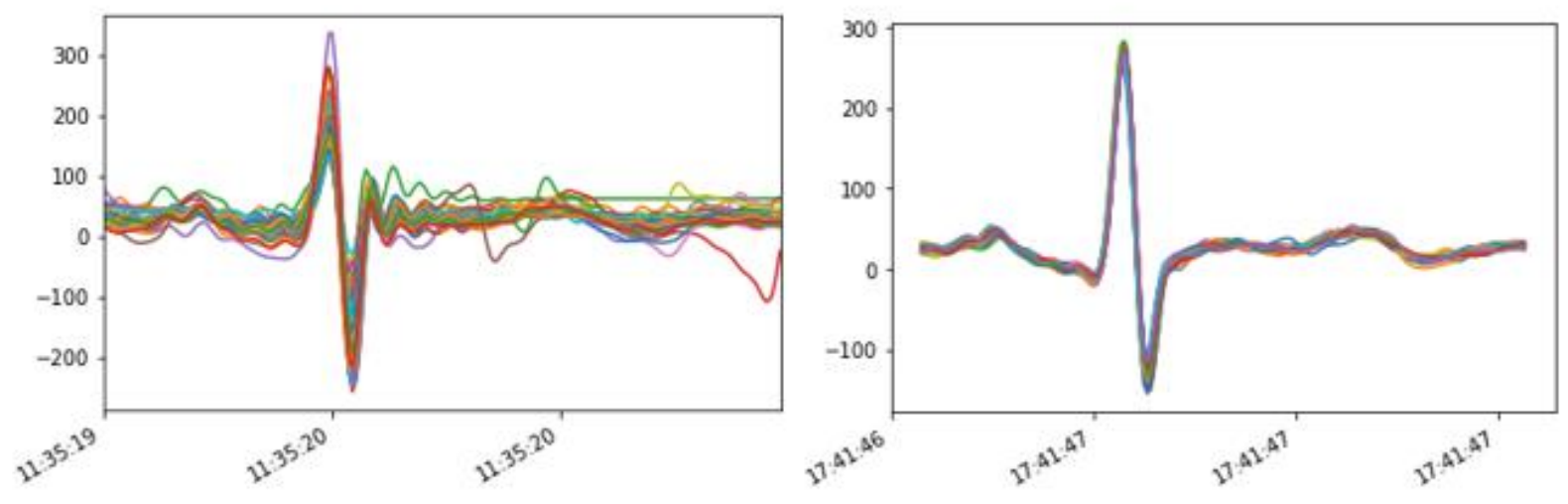

Figure 4 Cardiac Cycle Signal a) When loosely connected b) When Person in Resting Position

This score depends on the signal quality and also the cardiac cycle signal. In the Figure 4.a we have the low cardiac cycle signal. It is the cardiac cycle signal of the data from a signal with movement in the electrodes. It resulted in the class of Others with 54\% certainty as it deviates a major part from the general ECG signal values. The Figure 4.b we have a cardiac cycle signal with a quality of $99 \%$, this data is classified as Normal class with a certainty of $97.7 \%$.

\section{CONCLUSION}

This project comes up with a new way of implementation in the process of analyzing the real-time ECG readings that are acquired from a patient and results the class in which these readings come under. This results come under either in any one of the classes namely, AF, Normal, Other Signal, Disturbance. This project mainly counters the inability to track, if there is any unidentified state of the heartbeat. It involves the latest technological advancements like the implementation of Neurokit for feature extraction and analyzing the feature identified using CNN Algorithm. The training data that is used in the training model is obtained from Physionet Challenge 2017. By introducing the IoT device it helps the system by providing the input data dynamically unlike the previous models that used the pre-recorded data for analysis. The accuracy of the signal captured is in a range of $75-80 \%$ which defines the quality of the ECG signal. Although, it helps the person to identify if there is any need to attend a doctor checkup, it does not provide the accurate state of the heart that is not suitable for diagnosis purpose.

\section{FUTURE ENHANCEMENT}

The device is limited to personal use. The main advancement that is achieved in this project compared to previous implementations is the ECG data can be collected from the patient dynamically and process it to get the results. This project have very limited manual intervention like in the phase of starting the device and unlike the previous projects, just by initializing the process, the whole process runs dynamically in this project which limits the human intervention. The project has become limited to offline implementation. It can be carry forward as a funded real-time project by implementing the process on online platforms using the cloud services like AWS and by implementing the
Neurokit and the CNN Deep Learning Algorithm on this online cloud platform. This can also include many other features like a GUI for making it more user-friendly, develop a device that monitor the patient all the time with the support of a battery and add extra features like alerting the concerned hospital management and the patient's guardian if there is anything is suspicious about the patient's health condition.

\section{REFERENCES}

1. Amir-Mohammad Rahmani, Nanda Kumar Thanigaivelan, Tuan Nguyen Gia, Jose Granados, ehailu Negash, Pasi Liljeberg, and Hannu Tenhunen, "Smart e-Health Gateway: Bringing Intelligence to Internet-of-Things Based Ubiquitous Healthcare Systems", IEEE Consumer Communications and Networking Conference(CCNC), 2015.

2. Chao Lia, Xiangpei Hua, Lili Zhang, "The IoT-based heart disease monitoring system for pervasive healthcare service", International Conference on Knowledge Based and Intelligent Information and Engineering Systems, 2017

3. Fernando Andreotti, Oliver Carr, Marco A. F. Pimentel, Adam Mahdi, Maarten De Vos, "Comparing Feature Based Classifiers and Convolutional Neural Networks to Detect Arrhythmia from Short Segments of ECG", Conference, Computing in Cardiology, 2017

4. Alka S. Barhatte, Rajesh Ghongade, Abhishek S. Thakare, "QRS complex detection and arrhythmia classification using SVM", IEEE, Communication Control and Intelligent Systems (CCIS), 2015

5. S. S. Mehta, N. S. Lingayat, Member, IAENG, "Support Vector Machine for Cardiac Beat Detection in Single Lead Electrocardiogram ",IAENG International Journal of Applied Mathematics, 2007

\section{AUTHORS PROFILE}

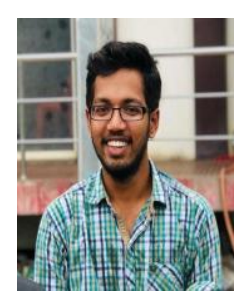

A Chandrahas Reddy currently pursuing his B.Tech, $4^{\text {th }}$ year in Department of Information Technology at SRM Institute of Science and Technology. His area of interest is Cryptography, Networking, Cyber Forensics and Data Analytics. Implemented projects in IoT and Networking. He is an avid reader of scientific and Computer related journals. His aim is to contribute himself to the scientific world. 


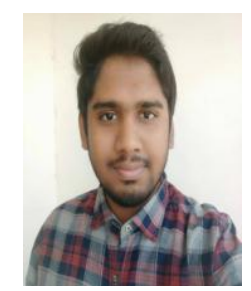

G Avinash Reddy currently pursuing his B.Tech $4^{\text {th }}$ year in Department of Information Technology from SRM Institute of Science and Technology. His area of interest is Cryptography, IOT, Security and Software Development. He implemented projects like Remote Water quality analysis using IOT, Aadhar based online voting machine.

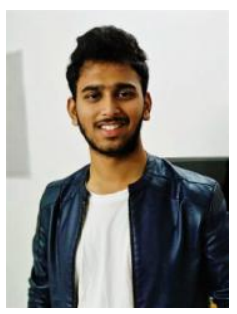

A V R M Koushik currently pursuing his B.Tech, $4^{\text {th }}$ year in Department of Information Technology at SRM Institute of Science and Technology. He is keen on the works which involve implementation of cryptography and security in the areas of IoT and web development. He has zeal to learn new things and interested in managing things.

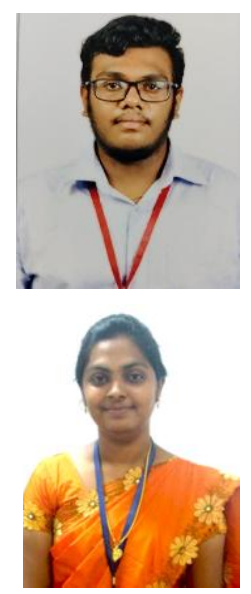

K V Siddeshwar currently pursuing his B.Tech, $4^{\text {th }}$ year in Department of Information Technology at SRM Institute of Science and Technology. His area of interest is Data Analytics , Product Development , Backend Development (SQL). Implemented projects in IoT and Data Visualisation.

Ms.C Fancy completed her B. Tech in IT and $\mathrm{ME}$,in Computer Science Engineering. She is working in the department of Information Technology, SRM Institute of Science and Technology as an Assistant Professor since 2013. She is currently pursuing Phd in the SDN Domain. Her research interests include cryptography, medical imaging and cyber security.

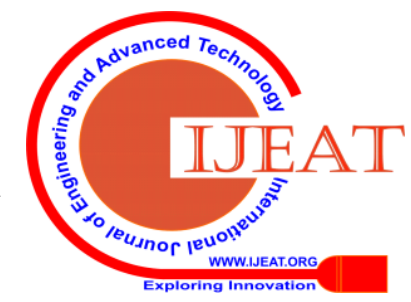

